\title{
Synchrotron Radiation Nanoscale X-ray Imaging Technology And Scientific Big Data Mining Assist Energy Materials Research
}

Kai zhang* ${ }^{1}$, Fang Ren ${ }^{2}$, Xuelong Wang ${ }^{3}$, Enyuan $\mathrm{Hu}^{3}$, Xiao-Qing Yang ${ }^{3}$, Yahong Xu ${ }^{2}$, Liquan Chen $^{4}$, Hong $\mathrm{Li}^{4}$, Piero Pianetta ${ }^{2}$, Apurva Mehta ${ }^{2}$, Xiqian $\mathrm{Yu}^{4}$ and Yijin Liu ${ }^{2}$

1.Beijing Synchrotron Radiation Facility, Institute of High Energy Physics, Chinese Academy of Sciences, Beijing and China

2.Stanford Synchrotron Radiation Light source, Palo Alto and USA

3.Chemistry Division, Brookhaven National Laboratory, New York and USA

4.Beijing National Laboratory for Condensed Matter Physics, Institute of Physics, Beijing and China

* Corresponding author: zhangk@ihep.ac.cn

The materials and devices we work with in modern society are often structurally complex and chemically heterogeneous. The complexity in the material is usually caused by the desired functionality that has requirements in many different aspects of the material properties. Taking Li-ion battery as an example, we often evaluate the device by combining several different characteristics, including the energy density, capacity, cyclability, temperature stability, price etc. As a result, material scientists need to look into the realistic systems, in which both the anticipated and the unanticipated material phases/reactions occur.

The importance of locating functionally important minority phases in obtaining a deeper understanding of complex working materials and devices has driven the rapid development of fast, high- resolution, chemically sensitive probes, especially at large scale experimental facilities such as synchrotrons. While the experimental capability is being greatly advanced, more effort is needed for the development of computing methods that could effectively and efficiently extract the scientifically important information from the large datasets.

A international research team led by Beijing Synchrotron Radiation Facility , Stanford Synchrotron Radiation Lightsource ,Institute of Physics (Beijing), and Brookhaven National Laboratory, applied the big data mining concept to the in-situ study of LiCoO2 battery electrodes. In a paper published earlier this year [1], this team demonstrated the in-situ monitoring of a selected LiCoO2 particle over many electrochemical cycles. Their results in that paper suggested that the particle is capable of readjusting itself in response to different local chemical environments (Figure 1).

While it is exciting to visualize the individual particle's behavior over long term cycling, scientists will naturally question the representativeness of a single particle to the entire battery cell. To address this question, the team surveyed the battery cell at many different locations. After initial data reduction, they effectively retrieved over 10 million x-ray absorption spectrum covering more than 100 active particles. They developed algorithm was used to convert the spectrum information into 2D image information for extraction of spectroscopic data attributes, which are then fed into the computing engine for clustering. The developed computing method identified two different particles that are abnormal in 
terms of their spectroscopic fingerprints. These two particles were further attributed to two different unwanted side reactions that happened during the electrochemical cycling (Figure2). This work is recently published in Nano Letters [2].

While the combination of in-situ nano imaging capability and the big data mining method has provided very useful insights in the field of battery research as presented in the current paper, it is anticipated that the development in data mining method will play an even more important role as the instrument/facility continues to advance.

By combining in-situ nano imaging capabilities and big data mining methods, this research has provided very useful insights in the field of battery research. Developments in data mining methods will play an even more important role as the facility continues to advance.

\section{References:}

[1]Yahong Xu, et al,, ACS Energy Lett. 2, (2017), 1240-1245.

[2]Kai Zhang et al, Nano Lett., 12 (2017), pp 7782-7788

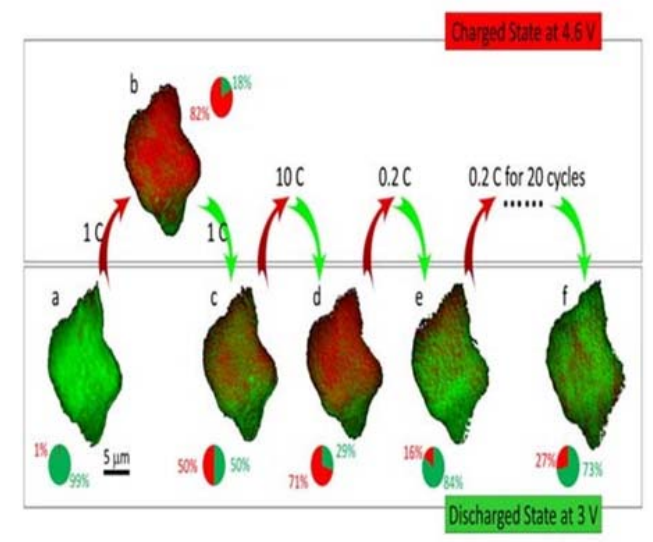

Figure 1. In situ monitoring of the chemical heterogeneity in a single particle of $\mathrm{LiCoO}_{2}$ up to 23 cycles at different cycling rates. The red area represents the domain at charged (oxidized, $\mathrm{Co} 4+$ ) state" and the green area represents the domain at discharged (reduced, Co3+) state. [ACS Energy Lett. 2, 1240-1245 (2017)]

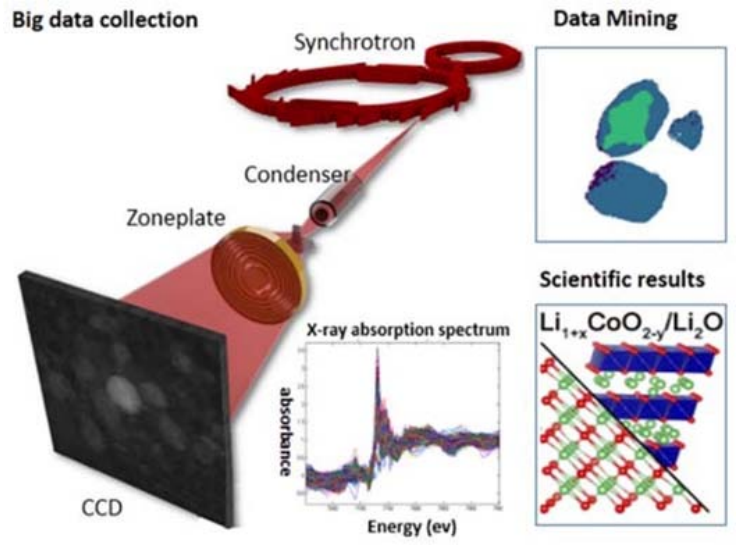

Figure 2. Big Data collection: Nanoscale X-ray spectro-microscopy technique (beamline 6-2C of SSRL) collects spatially resolved x-ray absorption spectra at very fast data rate. Data Mining: Advanced data mining approach developed in this work searches through over 10 million spectra and identified two particles with unanticipated chemical fingerprints. Scientific results: These two particles are attributed to two different unwanted side reactions.[ Nano Lett., 12 (2017), pp 7782-7788]. 